\title{
Factors Influencing Threshold Energy in Subthreshold Photocoagulation for Diabetic Macular Edema
}

\author{
Hirotsugu Takashina' \\ Akira Watanabe ${ }^{2}$ \\ Koji Komatsu' \\ Tadashi Nakano (iD) ${ }^{2}$ \\ 'Department of Ophthalmology, Tokyo \\ Rosai Hospital, Tokyo, Japan; \\ ${ }^{2}$ Department of Ophthalmology, The Jikei \\ University School of Medicine, Tokyo, \\ Japan
}

Background: Subthreshold photocoagulation is one of the treatments for diabetic macular edema. However, the range of adequate laser energy that can be used for subthreshold photocoagulation is very restricted. Therefore, determination of the titration settings for the threshold energy is an essential part of the subthreshold-photocoagulation procedure. This study examined factors influencing the threshold energy used in subthreshold photocoagulation for diabetic macular edema.

Methods: For 20 consecutive cases (29 eyes) who had received treatment using Endpoint Management (EpM) between April 2019 and June 2020 for persistent diabetic macular edema after completion of panretinal photocoagulation, multiple regression analysis was used to examine factors influencing threshold energy.

Results: Analysis evaluating all the surgical procedures $(n=150)$ determined there was statistical significance for a history of vitrectomy and cataract surgery, central macular thickness, interval from completion of panretinal photocoagulation, superior $6 \mathrm{~mm}$ subfield, and nasal $6 \mathrm{~mm}$ subfield. Further analysis examined surgical procedures that had been done in patients with a history of vitrectomy and cataract surgery $(n=116)$. This analysis showed that central macular thickness, axial length, interval from completion of panretinal photocoagulation, and $\mathrm{HbA}_{1 \mathrm{c}}$ were all statistically significant factors.

Conclusion: Threshold energy for diabetic macular edema was significantly influenced by a history of vitrectomy and cataract surgery, central macular thickness, and interval between the completion of panretinal photocoagulation and initial EpM. Transparency of ocular media and intraocular inflammation were speculated to be associated with these results.

Keywords: diabetic macular edema, subthreshold photocoagulation, panretinal photocoagulation, vitrectomy, cataract surgery

\section{Introduction}

Persistent diabetic macular edema (DME) requires continuous therapeutic intervention. Treatments for DME include photocoagulation, anti-VEGF injections, triamcinolone acetonide injections, and vitrectomy, with photocoagulation the most common. However, due to its destructiveness, ${ }^{1}$ conventional photocoagulation, such as panretinal photocoagulation (PRP), is not suitable for continuous therapeutic interventions over a long duration. Conversely, compared to conventional photocoagulation, subthreshold photocoagulation, which uses lower irradiating energy, is more suitable, due to the lack of chorioretinal damage. ${ }^{2}$ Details on the mechanism of subthreshold photocoagulation have been previously reported. For example, Inagaki et al reported on the expression of heatshock protein, which may block activity of the apoptotic and inflammatory pathways that cause cellular damage. ${ }^{1}$ Kern et al examined the photothermal influence with regard to
Correspondence: Hirotsugu Takashina Department of Ophthalmology, Tokyo Rosai Hospital, 4-I3-2I Omori-Minami, Ota-ku, Tokyo, I43-00I3, Japan,

Tel +8I-3-3742-730I ext 8116

Fax +8I-3-3744-9310

Email two-shina@s7.dion.ne.jp 
Table I Multiple regression results for all surgical procedures $(n=150)$

\begin{tabular}{|c|c|c|c|}
\hline & Mean (range) & $\boldsymbol{F}$ & CC \\
\hline Threshold energy & $175.7 \pm 41.6(100-350)(\mathrm{mW})$ & - & - \\
\hline Age & $65.1 \pm 9.3(5 \mathrm{I}-80)$ (years) & 2.22 & -0.13 \\
\hline $\mathrm{HbA}_{\mathrm{Ic}}$ & $6.8 \pm 0.9(5.3-8.6)(\%)$ & 5.67 & 0.20 \\
\hline Interval from completion of PRP & $26.8 \pm 29.9(2-138)$ (months) & $11.29 *$ & -0.27 \\
\hline Central macular thickness & $378.7 \pm I I 8.0(206-75 \mathrm{I})(\mu \mathrm{m})$ & $14.33^{*}$ & 0.30 \\
\hline Upper $6 \mathrm{~mm}$ subfield & $326.4 \pm 56.1(250-449)(\mu \mathrm{m})$ & $11.14 *$ & -0.27 \\
\hline Nasal $6 \mathrm{~mm}$ subfield & $336.0 \pm 53.6(259-5 \mathrm{I} 2)(\mu \mathrm{m})$ & $7.57^{*}$ & 0.23 \\
\hline Lower $6 \mathrm{~mm}$ subfield & $302.0 \pm 59.0(195-512)(\mu \mathrm{m})$ & 1.57 & -0.11 \\
\hline Temporal $6 \mathrm{~mm}$ subfield & $307.9 \pm 55.8(218-444)(\mu \mathrm{m})$ & 0.18 & 0.04 \\
\hline HVC & - & $36.76 *$ & -0.46 \\
\hline
\end{tabular}

Notes: $* P<0.01$

Abbreviations: PRP, panretinal photocoagulation; HVC, history of vitrectomy and cataract surgery; CC, correlation coefficient.

heat shock-protein expression and cell death. ${ }^{3}$ Since excessive laser energy causes destructive damage to the retina and moderate laser energy does not lead to the required therapeutic effect, the range of adequate laser energy that can be used for subthreshold photocoagulation is very restricted. Therefore, decisions on the necessary titration of the threshold energy are very important when performing the subthresholdphotocoagulation procedure. The present study examined factors that influence threshold energy in subthreshold photocoagulation for DME.

\section{Methods}

This retrospective study was approved by the Ethics Committee of the Tokyo Rosai Hospital (IRB 02-11, September 23, 2020), and complied with the tenets of the Declaration of Helsinki. Written informed consent was obtained from each patient. We evaluated 20 consecutive cases (29 eyes) who had received treatment using Endpoint Management (EpM), a subthreshold-photocoagulation procedure using a PASCAL laser (Topcon Medical Laser Systems, Santa Clara, CA, USA), for persistent DME after completion of PRP between April 2019 and June 2020. All eyes were performed using only a macular grid pattern. Inclusion criteria were no existence or surgical management of proliferative membrane, no vitreous hemorrhage and/or preretinal hemorrhage, and stable and being treated for diabetes by a physician. Exclusion criteria were receipt of either vitrectomy or cataract surgery only and a history of other vitreoretinal disease, uveitis, glaucoma, or corneal disease. Titration was performed in an area without retinal edema outside the vascular arcade, with the threshold energy determined by the appearance of a "barely visible" lesion within 3 seconds after the irradiation. Factors influencing threshold energy were examined using multiple regression analysis. The criterion variable for the multiple regression analysis of all surgical procedures was threshold energy, while age, $\mathrm{HbA}_{1 \mathrm{c}}$, interval from the completion of PRP, central macular thickness (CMT), retinal thickness of each $6 \mathrm{~mm}$ subfield (superior, nasal, inferior, temporal), and a history of vitrectomy and cataract surgery (HVC) were used as the independent variables. Subsequently, we performed a multiple regression analysis of the surgical procedures with HVC alone, with axial length added as an independent variable. Measurements of the CMT and retinal thickness of each $6 \mathrm{~mm}$ subfield were performed using optical coherence tomography (Triton Plus; Topcon). Differences with $p<0.01$ were considered statistically significant.

\section{Results}

The characteristics of DME in this study included spongelike retinal swelling and/or cystoid ME (CME), with none of the OCT images showing any serous retinal detachment. In the analysis of all of the surgical procedures $(n=150)$, the mean number of times that EpM was performed in each eye was $5.2 \pm 2.5$ (one to 12) times, and the mean, $F$-value, and correlation coefficient for each variable are shown in Table 1. HVC ( $F=36.76$, negative correlation), CMT ( $F=14.33$, positive correlation), interval from completion of PRP ( $F=11.29$, negative correlation), superior 
Table 2 Multiple regression analysis results for surgical procedures with HVC $(n=116)$

\begin{tabular}{|c|c|c|c|}
\hline & Mean (range) & $\boldsymbol{F}$ & CC \\
\hline Threshold energy & $164.4 \pm 34.2(100-250)(\mathrm{mW})$ & - & - \\
\hline Age & $64.9 \pm 10.3(5 \mid-80)$ (years) & 2.54 & 0.15 \\
\hline $\mathrm{HbA}_{\mathrm{Ic}}$ & $6.6 \pm 0.7(5.3-8.4)(\%)$ & $10.02 *$ & 0.29 \\
\hline Interval from completion of PRP & $25.3 \pm 32.4$ (2-138) (months) & $10.39 *$ & -0.30 \\
\hline Central macular thickness & $389 . \pm 109.0(206-75 \mathrm{I})(\mu \mathrm{m})$ & $14.85^{*}$ & 0.35 \\
\hline Upper $6 \mathrm{~mm}$ subfield & $321.1 \pm 50.9(250-445)(\mu \mathrm{m})$ & 0.05 & -0.02 \\
\hline Nasal $6 \mathrm{~mm}$ subfield & $325.4 \pm 45.6(259-453)(\mu \mathrm{m})$ & 0.98 & 0.10 \\
\hline Lower $6 \mathrm{~mm}$ subfield & $287.2 \pm 42.9(195-388)(\mu \mathrm{m})$ & 6.36 & 0.24 \\
\hline Temporal $6 \mathrm{~mm}$ subfield & $299.8 \pm 56.7(218-444)(\mu \mathrm{m})$ & 4.21 & -0.20 \\
\hline Axial length & $23.88 \pm 1.29(22.17-27.43)(\mathrm{mm})$ & $12.77^{*}$ & 0.33 \\
\hline
\end{tabular}

Notes: $P<0.01$.

Abbreviations: PRP, panretinal photocoagulation; HVC, history of vitrectomy and cataract surgery; CC, correlation coefficient.

$6 \mathrm{~mm}$ subfield ( $F=11.14$, negative correlation), and nasal $6 \mathrm{~mm}$ subfield ( $F=7.57$, positive correlation) were all statistically significant. In the analysis of the surgical procedures with HVC $(n=116)$, the mean number of of EpMs in each eye was $5.3 \pm 2.1$ (two to eight). The mean, $F$-value, and correlation coefficient for each of the variables are shown in Table 2. CMT ( $F=14.85$, positive correlation), axial length ( $F=12.8$, positive correlation), interval from completion of PRP ( $F=10.39$, negative correlation), and $\mathrm{HbA}_{1 \mathrm{c}}(F=10.02$, positive correlation) were all statistically significant.

\section{Discussion}

The presence of HVC decreased the threshold energy, as indicated by the negative correlations in the analysis of all the surgical procedures. Furthermore, as the $F$-value for HVC was the largest of all the independent variables, HVC was likely the most influential factor in the determination of threshold energy. If satisfactory transparency of the ocular media is present due to HVC, there should be a slight loss of the laser energy in ocular media, and this should assist the surgeons when dealing with the presence of a barely visible lesion. Therefore, HVC was thought to be one of the significant factors in the present study. Lavinsky et al suggested that visibility of retinal lesions might depend on transparency of ocular media due to subjectivity, ${ }^{2}$ and the result in the present study backed this up statistically. On the other hand, retinal thickness at the titration area might have been another factor influencing threshold energy, because of the area where the titration procedure was actually performed. Unfortunately, this could not be used as an independent variable in the present study. In contrast, we supposed there was no significance found for CMT or each $6 \mathrm{~mm}$ subfield, because these was not titration area. However, a few variables were statistically significant. With the exception of HVC, CMT was the most influential factor, with the largest $F$-values on both analyses. Moreover, the positive correlation of CMT on both analyses indicated that thicker CMT required higher threshold energy. We speculated that the unique pathology observed in CME might be responsible for this paradox, regardless of CMT itself. Therefore, even if retinal edema near the fovea does not exist, CME can occur due to intraocular inflammation, such as uveitis. As a result, intraocular inflammation in diabetic retinopathy might cause thickening of the retina at the titration area and the fovea.

Though analysis of all surgical procedures showed significance for the upper and nasal $6 \mathrm{~mm}$ subfields, the correlation coefficients were opposing (upper subfield negative, nasal subfield positive), and there was no significance found for any of the $6 \mathrm{~mm}$ subfields on analysis of surgical procedures with HVC. There is normally sufficient distance between the $6 \mathrm{~mm}$ subfield and titration area (neighboring vascular arcade) compared to the diameter of the photocoagulation scar. Furthermore, the shape of DME is generally asymmetric in ophthalmology practice, and titration is normally performed in an area without any 
apparent retinal edema. For these reasons, we speculated that any significant correlation between retinal thickness for each $6 \mathrm{~mm}$ subfield and threshold energy might not actually exist. In contrast, both analyses showed that the shorter interval from completion of PRP resulted in higher threshold energy, shown by the negative correlation. Ascending VEGF levels immediately following $\mathrm{PRP}^{4}$ can lead to thickening of the whole retina, so we speculated this could potentially lead to increased threshold energy.

There were limitations in the present study. First, there was a difference in EpM repetitions inthe cases (two to eight), and no statistical correction was applied for both eyes of a single patient. These could have potentially influenced the detection of different factors between the analyses and thus might be the reason it was not possible to resolve the cause of the statistical significance for axial length in our analysis of surgical procedures with HVC. Second, $\mathrm{HbA}_{1 \mathrm{c}}$ values in both analyses were those at the initial EpM in each eye. Because there was stable diabetes treatment being administered by a physician, errors in $\mathrm{HbA}_{1 \mathrm{c}}$ throughout the duration of this study would likely have been restricted. Similarly, we could not include the duration of diabetes or renal function as general conditions, because we could not confirm data from the medical records. Third, we did not classify foveal pathology, such as macular atrophy. In conclusion, threshold energy for DME was significantly influenced by HVC, CMT, and the interval between the completion of PRP and initial EpM. It is our belief that transparency of ocular media and intraocular inflammation were responsible for this result.

\section{Acknowledgments}

The authors thank the orthoptists at Tokyo Rosai Hospital, Hiroyuki Naruke, Yuka Yanagisawa, and Yuka Kobayashi, for helpful discussions.

\section{Disclosure}

The authors report no conflicts of interest in this work.

\section{References}

1. Inagaki K, Shuo T, Katakura K, Ebihara N, Murakami A, Ohkoshi K. Sublethal photothermal stimulation with a micropulse laser induces heat shock protein expression in ARPE-19 cells. J Ophthalmol. 2015;2015:729792. doi:10.1155/2015/729792

2. Lavinsky D, Sramek C, Wang J, et al. Subvisible retinal laser therapy: titration algorithm and tissue response. Retina. 2014;34:87-97. doi:10.1097/IAE.0b013e3182993edc

3. Kern K, Mertineit CL, Brinkmann R, Miura Y. Expression of heat shock protein 70 and cell death kinetics after different thermal impacts on cultured retinal pigment epithelial cells. Exp Eye Res. 2018;170:117-126. doi:10.1016/j.exer.2018.02.013

4. Itaya M, Sakurai E, Nozaki M, et al. Upregulation of VEGF in murine retina via monocyte recruitment after retinal scatter laser photocoagulation. Investig Ophthalmol Vis Sci. 2007;48:5677-5683. doi:10.1167/iovs.07-0156
Clinical Ophthalmology

\section{Publish your work in this journal}

Clinical Ophthalmology is an international, peer-reviewed journal covering all subspecialties within ophthalmology. Key topics include: Optometry; Visual science; Pharmacology and drug therapy in eye diseases; Basic Sciences; Primary and Secondary eye care; Patient Safety and Quality of Care Improvements. This journal is indexed on PubMed

\section{Dovepress}

Central and CAS, and is the official journal of The Society of Clinical Ophthalmology (SCO). The manuscript management system is completely online and includes a very quick and fair peer-review system, which is all easy to use. Visit http://www.dovepress.com/ testimonials.php to read real quotes from published authors. 